\title{
DOPPLER STUDY OF THE UTERINE AND UMBILICAL ARTERY TO PREDICT INTRAUTERINE GROWTH RETARDATION
}

\begin{tabular}{|l|l|l|l|}
\hline Radiodiagnosis &
\end{tabular}

Dr.Rabiya Baseri Assistant Professor, Department of Radiodiagnosis, Alluri Sita Ramaraju Academy of Nelofar*

Dr.Sushma Vuyyuru

\section{Dr.Sri Hari Raavi}

\section{Dr.Nandam Hema} Mohana Lakshmi

\begin{abstract}
The primary objective of this study is to transvalue the role of colour doppler sonography in the evaluation of pregnancy with intrauterine growth restriction. The study included 50 antenatal women with singleton pregnancy diagnosed as having a fetus with intrauterine growth restriction based on greyscale ultrasound findings. Further, an obstetric Doppler Ultrasound was done, and diagnostic statistics were applied to determine the Umbilical artery PI, RI, and Uterine artery PI, RI and correlating with perinatal outcomes. The specificity, sensitivity, positive predictive value, negative predictive value, and diagnostic accuracy were determined for all Doppler measurements. Among women with an identifiable cause, $42 \%$ had pregnancy-induced hypertension (PIH), $40 \%$ had moderate to severe anaemia complicating pregnancy. At the same time, $20 \%$ of the study group had no detectable cause for IUGR. The persistence of early diastolic notch beyond 26 weeks showed $60 \%$ sensitivity as a predictor for the perinatal outcome. The sensitivity of RI, PI of the uterine artery in predicting perinatal outcomes was $73 \%$ and $76.7 \%$, respectively. Whereas, the sensitivity of RI, PI of the Umbilical artery in predicting perinatal outcomes was $63 \%$ and $70 \%$, respectively. Doppler imaging provides indirect evidence of fetal compromise and is known to improve outcomes of high-risk pregnancies with intrauterine growth restriction. Hence, it is of eminent value for monitoring during the pregnancy.
\end{abstract}

\section{KEYWORDS}

\section{INTRODUCTION}

The term Intrauterine growth restriction (IUGR) is used to describe the condition of a fetus whose size or growth is subnormal. The most common definition of intrauterine growth restriction is : a fetus is growth restricted if its weight is less than the tenth percentile for its gestational age

IUGR has many causes; maternal causes are hypertension, renal disease, collagen vascular disease, drug or alcohol abuse and poor nutrition. In utero fetal infections such as cytomegalovirus, toxoplasmosis and chromosomal anomalies such as triploidy and trisomies 13 and 18 also result in IUGR.2 Placental insufficiency in the absence of any cause is called primary placental insufficiency and is a common cause of IUGR.

Correct antenatal diagnosis of IUGR by real-time ultrasound can reduce the complications and improve the perinatal outcome. With the introduction of ultrasound, the small fetuses could be identified. However, it could not be possible to identify which of these fetuses were at increased risk due to uteroplacental insufficiency. Therefore, the non-stress test and the biophysical profile are used to monitor small fetuses.

Doppler ultrasound evaluation of fetal circulation non-invasively assessed the fetal response to hypoxia by evaluating the uteroplacental and fetoplacental blood flow. Thus it became possible to identify those small fetuses that were at increased risk of perinatal morbidity and mortality due to hampered uteroplacental and fetoplacental circulations.

The present study focusses on establishing the role of Umbilical artery and Uterine artery Doppler ultrasonography in predicting poor perinatal outcomes in clinically suspected IUGR pregnancies and on determining the role of Doppler velocimetry in the clinical management of such pregnancies.

\section{OBJECTIVES}

- To evaluate the role of colour Doppler sonography in pregnancies with intrauterine growth restriction by assessing the uteroplacental and fetoplacental circulations.
- To evaluate the usefulness of Uterine artery and Umbilical artery Doppler indices as predictors of adverse perinatal outcome in clinically suspected IUGR pregnancies.

- To determine the role of Doppler ultrasound in the management of IUGR pregnancy.

\section{RESULTS}

A prospective study on 50 antenatal women diagnosed as having a fetus with intrauterine growth restriction based on greyscale ultrasound findings. Flow velocity waveforms of the Uterine and Umbilical arteries were obtained from all the 50 cases and were analyzed.

\section{DOPPLER STUDIES:}

In the present study, R.I and P.I Doppler indices of both the Uterine and Umbilical arteries were taken according to the 50 th percentile.

\section{UTERINE ARTERY DOPPLER STUDIES}

1.CHANGES IN UTERINE ARTERY RESISTANCE INDEX (RI)

Thirty mothers $(60 \%)$ had an elevated Uterine artery RI, and 20 patients (40\%) had normal Uterine artery RI.

\section{CHANGES IN UTERINE ARTERY PULSATILITY INDEX (PI)}

Thirty-three mothers $(66 \%)$ had an elevated Uterine artery PI, and 17 patients (34\%) had normal Uterine artery PI.

\section{PERSISTENT EARLY DIASTOLIC NOTCH OF UTERINE ARTERY}

In the present study, the diastolic notch was present in 18 patients(36\%), out of which 28 per cent had notches in bilateral arteries, and 8 per cent had notch involving single artery. Thirty-two patients $(64 \%)$ had normal Uterine artery flow waveform.

CHANGES IN UTERINE ARTERY USING THREE PARAMETERS(RI, PI\& DIASTOLIC NOTCH)

$66 \%$ of antenatal mothers with IUGR showed an abnormal Uterine artery flow velocity profile when all three parameters ( RI, PI and presence of persistent early diastolic notch) were considered. The rest 
of the $34 \%$ showed normal uterine artery velocity profiles.

\section{UMBILICALARTERY DOPPLER STUDIES}

\section{UMBILICALARTERY PULSATILITY INDEX}

27 mothers (54\%) had an elevated Umbilical artery PI, and 23 patients $(46 \%)$ had standard Umbilical artery PI.

\section{UMBILICALARTERY RESISTANCE INDEX}

Twenty-five mothers (50\%) had an elevated Umbilical artery RI, and 25 patients $(50 \%)$ had normal Umbilical artery RI

\section{UMBILICAL ARTERY END DIASTOLIC FLOW PATTERN}

Eleven fetuses (22\%) showed absence end-diastolic flow and no fetus had a reversal of end-diastolic Umbilical artery flow. Rest of the thirtynine fetuses had a positive diastolic flow.

CHANGES IN UMBILICAL ARTERY USING THREE PARAMETERS(RI, PI \&END DIASTOLIC FLOW PATTERN)

Twenty-seven patients(54\%) had an abnormal Umbilical artery flow velocity profile when all three parameters ( RI, PI and end-diastolic flow pattern) were considered. The rest of the $23 \operatorname{cases}(46 \%)$ showed standard umbilical artery velocity profiles.

\section{ANALYSIS OF BOTH UTERINE AND UMBILICAL ARTERIES}

$82 \%$ of the study group (41 mothers) showed abnormalities in either the Uterine or Umbilical arteries when both were assessed together. When individual arteries were considered, Uterine arteries showed better pick up rates (66\%) than the Umbilical arteries (54\%).

\section{ANALYSIS OF PERINATAL OUTCOME}

There were five intrauterine deaths and 45 live births. Of the 45 live births, ten neonates were admitted to NICU, 22 neonates had 5 min APGAR score of less than 7 , and 23 babies were born by emergency caesarian section. There were 30 neonates with birth weight less than $2.5 \mathrm{~kg}$. Thirty fetuses $(60 \%) \mathrm{had}$ at least one adverse perinatal outcome. The remaining 20 fetuses $(40 \%)$ had a favourable outcome.

\section{PERINATAL MORTALITY V/S ABSENT END-DIASTOLIC FLOW}

Absent end-diastolic flow velocity was seen in 11 fetuses (22\%) in our study, of which 5 were Intra Uterine deaths. The mortality in cases of absent end-diastolic flow was $45 \%$

\section{REPRESENTATIVE CASE}

CASE: A 32-year-old gravid mother with 31 weeks of gestation showed the following Doppler indices

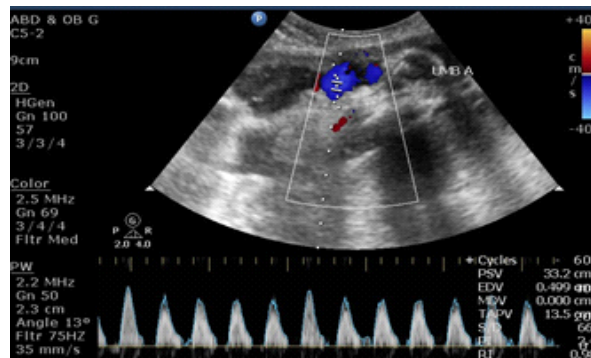

Figure 1: arrow pointing to absent end diastolic flow

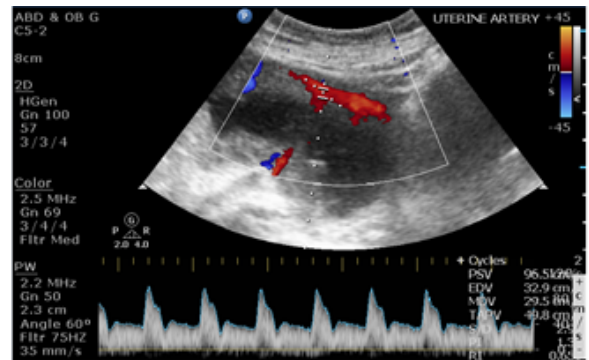

Figure 2: arrow pointing to persistent early diastolic notch

\section{DISCUSSION}

Intrauterine growth restriction is merely a result of a series of events occurring along several possible pathways. Hence, an accurate antenatal diagnosis must decide whether the fetus is constitutionally small for gestational age or small as a consequence of impaired placental perfusion. Analysis of Doppler flow velocities can solve this problem by examining Uterine arteries (uteroplacental circulation) and Umbilical arteries (fetoplacental circulation).

\section{Etiology of IUGR}

Among women where a cause for IUGR was identified, $42 \%$ had pregnancy-induced hypertension (PIH), 40\% had moderate to severe anaemia complicating pregnancy. This can be attributed to asymmetrical IUGR, which accounts for $80 \% .20 \%$ of the study group had no detectable cause for IUGR

\section{Uterine artery}

In the present study, the persistent early diastolic notch beyond 26 weeks of gestation showed a sensitivity of $60 \%$.

The sensitivity of RI was $73 \%$ in our study in comparison with that of Benson and Doubliet3 (67\%) and Coleman et al4(83\%). This discrepancy may be attributed to different cut off levels of the resistive index, varying between 0.5 to 0.62 .

The sensitivity of PI was $76.7 \%$ in our study in comparison with that of Lavanya Rai et al5(86\%) and BN Lakhkar et al6(83\%). This discrepancy may be attributed to different cut off levels of pulsatility index, varying between $0.6-0.8$

The specificity of Uterine artery Doppler study to detect adverse perinatal outcome was $60 \%$ with Uterine artery RI and 50\% with Uterine artery PI

When these Doppler parameters were taken into account, the sensitivity of Uterine artery Doppler for predicting perinatal outcome was high. This is in agreement with the study by Zimmermann et al7, who found a combination of several Doppler parameters to be superior to a single parameter.

\section{Umbilical artery}

The umbilical artery was the primary vessel used for the monitoring of high-risk pregnancies as the umbilical artery represents fetoplacental circulation and reflects placental resistance. In the present study, the Umbilical artery PI had a sensitivity of $70 \%$, and Umbilical artery RI had a sensitivity of $63 \%$ in predicting perinatal outcome. The specificity of Doppler examination of the Umbilical artery using PI in predicting perinatal outcome was $70 \%$ and with RI was $70 \%$.

Absent or reversal of the end-diastolic flow velocity was seen in $22 \%$ fetuses in our study compared with that of $37 \%$ in the study by Benson and Doubilet ${ }^{3}$.

Doppler studies of the Umbilical artery and Uterine artery together had better sensitivity than that of the individual vessels. However, among these, Uterine artery Doppler indices showed more sensitivity, and Umbilical artery Doppler indices showed more specificity.

\section{Prediction of perinatal outcome}

Thirty fetuses had at least one adverse outcome. Remaining 20 fetuses had a favorable outcome. There were 5 intrauterine deaths and 45 live births. Of the 5 IUD's, all had absent diastolic flow. The mortality in cases of absent end diastolic flow was $45 \%$, indicating grave prognosis. Of the 45 live births, 10 neonates were admitted to NICU, 22 neonates had 5 min APGAR score less than 7, and 23 babies were born by emergency caesarian section. These results are slightly higher than Gramellini et al64. This can be contributed to the difference in perinatal mortality and morbidity rates from western standards to Indian standards.

\section{CONCLUSION}

The Umbilical-placental and Uterine vascular beds are directly involved in the hemodynamic adjustments of fetal growth restriction. A Doppler index that reflects both of these areas can be useful for identifying fetuses with increased uteroplacental and fetoplacental circulations.

Assessment of uteroplacental and fetoplacental circulations together is more sensitive in the prediction of perinatal outcomes.

Doppler imaging is of value for monitoring the pregnancy because it can provide indirect evidence of fetal compromise and is known to 
improve outcomes of high-risk pregnancies with intrauterine growth restriction. Hence Doppler evaluation is complementary to all other surveillance modalities.

\section{REFERENCES:}

Lugo G.Cassady. Intrauterine growth retardation clinicopathological findings in 233 consecutive infants. Am J Obstet Gynecol 1971; 109: 615-122.

2. Seeds JW Impaired fetal growth: definition and clinical diagnosis Obstet Gynecol 1984; 64:303-310.

3. Benson $\mathrm{CB}$, Doubilet PM. Doppler criteria for intrauterine growth retardation: predictive values. J Ultrasound Med. 1988; 7: 655-659.

4. Coleman MA, McCowan LM, North RA. Mid-trimester Uterine artery Doppler screening as a predictor of adverse pregnancy outcome in high-risk women. Ultrasound Obstet Gynecol 2000; 15 (1): 4-6.

5. Lavanya Rai, Lakshmi S. Value of Third Trimester Uterine Artery Doppler in High Risk pregnancies for prediction of adverse perinatal outcome. South Asian Federation of pregnancies for prediction of adverse perin
Obstetrics and Gynaecology 2010;2(1):31-35.

6. Bhushan N Lakhkar, Shefeek A Ahamed. Doppler velocimetry of Uterine and Umbilical arteries during pregnancy. Indian Journal Of Radiology and Imaging 1999;9(3):119125.

7. Zimmermann P, Eirio V, Koskinen J, Kujansuu E, Ranta T. Doppler assessment of the Uterine and uteroplacental circulation in the second trimester in pregnancies at high risk for pre-eclampsia and/or intrauterine growth retardation: Comparison and correlation between different Doppler parameters. Ultrasound Obstet Gynecol. 1997; 9: 162-172. 